\title{
Erratum: Path-integral action of a particle with the generalized uncertainty principle and correspondence with noncommutativity [Phys. Rev. D 99, 104010 (2019)]
}

Sunandan Gangopadhyay and Sukanta Bhattacharyya

(Q) (Received 7 March 2020; accepted 12 March 2020; published 26 March 2020)

DOI: 10.1103/PhysRevD.101.069903

We note that there is an error in Eq. (40) in our paper. We give the correct expression here. In the paper we have formulated the path-integral representation of the transition amplitude of a particle moving in an arbitrary potential in the framework of the generalized uncertainty principle (GUP). From the path integral, we have obtained the action of the particle. We have also calculated the classical action for the harmonic oscillator in the presence of the GUP. We note that the classical action obtained in Eq. (40) in the paper is incorrect. This was also pointed out in [1]. Here we give the correct expression for the classical action $S_{c}(\beta)$ of the harmonic oscillator in the GUP framework to first order in the GUP parameter $\beta$ as

$$
\begin{aligned}
S_{c}(\beta)= & -\frac{1}{32} \beta m^{3} \omega^{3} \csc ^{4}(\omega T)\left[\left(q_{0}^{4}+q_{f}^{4}\right) \sin (4 \omega T)-44 q_{0} q_{f}\left(q_{0}^{2}+q_{f}^{2}\right) \sin (\omega T)-12 q_{0} q_{f}\left(q_{0}^{2}+q_{f}^{2}\right) \sin (3 \omega T)\right. \\
& +24 q_{0}^{2} q_{f}^{2} \omega T \cos (2 \omega T)-48 q_{0} q_{f} \omega T\left(q_{0}^{2}+q_{f}^{2}\right) \cos (\omega T)+12 \omega T\left(q_{0}^{4}+4 q_{0}^{2} q_{f}^{2}+q_{f}^{4}\right) \\
& \left.+4\left(2 q_{0}^{4}+15 q_{0}^{2} q_{f}^{2}+2 q_{f}^{4}\right) \sin (2 \omega T)\right] .
\end{aligned}
$$

This corrected form of the action does not change the conclusions drawn in our paper. One of the main results in the paper is Eq. (16).

[1] D. Park and E. Jung, Phys. Rev. D 101, 068501 (2020). 